\title{
An Improved Chaos Genetic Algorithm for T-Shaped MIMO Radar Antenna Array Optimization
}

\author{
Xin Fu, Xianzhong Chen, Qingwen Hou, Zhengpeng Wang, and Yixin Yin \\ School of Automation \& Electrical Engineering, University of Science and Technology Beijing, Beijing 100083, China \\ Correspondence should be addressed to Xin Fu; fxzn2006@163.com
}

Received 10 June 2014; Revised 3 September 2014; Accepted 9 September 2014; Published 14 October 2014

Academic Editor: Miguel Ferrando Bataller

Copyright ( 2014 Xin Fu et al. This is an open access article distributed under the Creative Commons Attribution License, which permits unrestricted use, distribution, and reproduction in any medium, provided the original work is properly cited.

\begin{abstract}
In view of the fact that the traditional genetic algorithm easily falls into local optimum in the late iterations, an improved chaos genetic algorithm employed chaos theory and genetic algorithm is presented to optimize the low side-lobe for T-shaped MIMO radar antenna array. The novel two-dimension Cat chaotic map has been put forward to produce its initial population, improving the diversity of individuals. The improved Tent map is presented for groups of individuals of a generation with chaos disturbance. Improved chaotic genetic algorithm optimization model is established. The algorithm presented in this paper not only improved the search precision, but also avoids effectively the problem of local convergence and prematurity. For MIMO radar, the improved chaos genetic algorithm proposed in this paper obtains lower side-lobe level through optimizing the exciting current amplitude. Simulation results show that the algorithm is feasible and effective. Its performance is superior to the traditional genetic algorithm.
\end{abstract}

\section{Introduction}

With the development of the MIMO communication technology, people put forward the concept of MIMO radar. MIMO radar antenna array pattern synthesis and optimization directly affect the performance of the whole radar system and performance. Low side-lobe optimization of antenna array is a typical multidimensional nonlinear optimization problem. In the given antenna array shape, number, and spacing of the premise, how to choose the feeding current amplitude and phase appropriately to minimize the peak side-lobe level is an important subject in the antenna array integrated.

In recent years, some intelligent optimization algorithm is more and more widely used in the antenna array pattern synthesis, such as neural network, quasi-Newton method, conjugate gradient method, simulated annealing, immune algorithm, and genetic algorithm, [1-8]. In addition, the method of Woodward, Dolph-Chebyshev polynomial method, Taylor method, Schelkunoff polynomial method, and so forth [911], only for uniform linear array and the same direction and spacing of the rectangular array, can realize low sidelobe pattern, but their application is quite limited. In [1217], the genetic algorithm with its excellent global search ability has been successfully widely applied in sparse antenna array, reducing the maximum side-lobe level and controlling array element position, phase, and amplitude of exciting current antenna array pattern synthesis. In [18-21], the GA was customized for adjusting the weight coefficients and the antenna positions simultaneously. But it can fall into local optimum easily.

Chaos initial value sensitivity, ergodicity, and regularity can be used for the optimization problem. It cannot only search efficiently but also avoid falling into local optimum. In recent years, many scholars put chaotic systems into the genetic algorithm and the optimization ability of genetic algorithm was improved greatly. In [22], the genetic algorithm using Logistic model to generate chaos sequence as initial population or add to the chaos in the mutation random disturbance, the algorithm performance is improved, but there are the big search for the blind area and the slow convergence speed. In [23], the probability density of chaotic sequence produced by Logistic mapping is in obedience to Chebyshev distribution which is seriously affect the global search ability and efficiency.

This paper puts forward the improved chaos genetic algorithm for T-shaped MIMO radar antenna array which 
has $M$ transmitting elements and $N$ receiving elements to optimize the feeding current amplitude (see Figure 4). Novel two-dimension Cat chaotic mapping has been proposed. We improve the Tent map and present the improved chaos genetic algorithm to reduce the side-lobe level of antenna array. The novel algorithm effectively solves the design problems of MIMO radar antenna pattern synthesis in low side-lobe level. The simulation results verify the effectiveness of the algorithm. The improved chaotic genetic algorithm has a good application prospect to solve the problem of antenna array.

\section{Analysis for T-Shaped MIMO Radar Antenna Array Pattern}

Consider a MIMO radar antenna array, which consists of $M$ transmitting antenna and $N$ receiving antenna evenly placed into T-shaped array (see as Figure 1).

The array element position, respectively, is described as

$$
r_{T}=\left[r_{T 1}, r_{T 2}, \ldots, r_{T M}\right], \quad r_{R}=\left[r_{R 1}, r_{R 2}, \ldots, r_{R N}\right]
$$

MIMO radar antenna pattern [24] can be equivalent to

$$
\begin{aligned}
F_{\mathrm{MIMO}}(\theta, \varphi)= & \left|\sum_{i=0}^{M-1} I_{T i} \exp \left[j k\left(r_{T i x} u+r_{T i y} v\right)\right]\right| \\
& \otimes\left|\sum_{i=0}^{N-1} I_{R i} \exp \left[j k\left(r_{\text {Rix }} u+r_{\text {Riy }} v\right)\right]\right|,
\end{aligned}
$$

where $k=2 \pi / \lambda$ and the coordinates of the $i$ th transmitting and receiving array element can be written as $\left(r_{\text {Tix }}, r_{\text {Tiy }}\right)$ and $\left(r_{\text {Rix }}, r_{\text {Riy }}\right)$. The normalized current amplitudes are $I_{T m}$ and $I_{R n}$, where $u=\sin \theta \cos \varphi, v=\sin \theta \sin \varphi, \theta$ and $\varphi$ denote the angle between the direction of array antenna far-field radiation plane wave and the normal direction of the array, and $\otimes$ denotes Kronecker product. We suppose that element spacing is $d_{x}=d_{y}$. Equation (2) can be described as

$$
\begin{aligned}
F(\theta, \varphi)= & \sum_{m=1}^{M} I_{T m} \exp \left\{j k\left[m-\frac{M+1}{2}\right] d_{x} u\right\} \\
& \cdot \sum_{n=1}^{N} I_{R n} \exp \left(j k n d_{y} v\right) .
\end{aligned}
$$

\section{Improved Chaos Genetic Algorithm}

3.1. Improved Tent Map. Tent map is a piecewise linear onedimensional mapping. The mathematical expression can be written as

$$
x_{n+1}=\alpha-1-\alpha\left|x_{n}\right|, \quad \alpha \in[1,2] .
$$

When $\alpha=2$, Tent map is known as the center Tent map. The expression of Tent map is defined by (5), whose variant

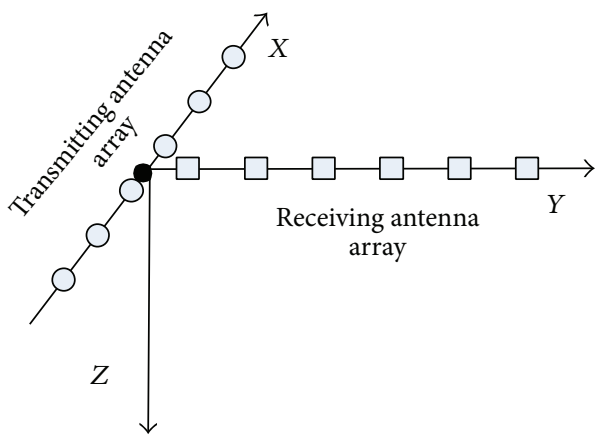

FIgURE 1: Distribution of T-shaped MIMO radar antenna array.

through Bernoulli shift transformation is shown by (6) [25]. Consider

$$
\begin{aligned}
& x_{k+1}= \begin{cases}2 x_{k}, & 0 \leq x_{k} \leq 0.5, \\
2\left(1-x_{k}\right), & 0.5<x_{k} \leq 1,\end{cases} \\
& k=0,1,2, \ldots, \\
& x_{k+1}= \begin{cases}2 x_{k}, & 0 \leq x_{k} \leq 0.5, \\
2 x_{k}-1, & 0.5<x_{k} \leq 1\end{cases} \\
& k=0,1,2, \ldots .
\end{aligned}
$$

Notice that (6) can be compressed into one representation; that is, $x_{k+1}=\left(2 x_{k}\right)$ mod1. We deployed random equation to improve Tent map. The chaos expression can be written as

$$
x_{k+1}=\left(2 * x_{k}\right) \bmod 1+\rho, \quad \rho \in(0,0.1) .
$$

Under the disturbance of random equation, Tent map can reachieve into the chaotic state at small cycle point or fixed point to enhance the ergodicity that can be better able to achieve global chaos optimization. Improved Tent map chaotic state distribution is shown as Figure 2.

3.2. The Cat Map. Two-dimensional Cat map [26], which is well-performing uniformity traversal, not easy to fall into the pulmonary circulation and fixed point, is introduced to chaos genetic algorithm. Two-dimensional Cat mapping equation is

$$
\begin{aligned}
& x_{n+1}=\left(x_{n}+y_{n}\right) \bmod 1, \\
& y_{n+1}=\left(x_{n}+2 y_{n}\right) \bmod 1 .
\end{aligned}
$$

That can be written as matrix form

$$
\left[\begin{array}{l}
x_{n+1} \\
y_{n+1}
\end{array}\right]=\left[\begin{array}{ll}
1 & 1 \\
1 & 2
\end{array}\right] \cdot\left[\begin{array}{l}
x_{n} \\
y_{n}
\end{array}\right] \bmod 1=C\left[\begin{array}{l}
x_{n} \\
y_{n}
\end{array}\right] \bmod 1,
$$

where $x \bmod 1=x-[x], C=\left[\begin{array}{ll}1 & 1 \\ 1 & 2\end{array}\right]$. "Mod" represents modulus after division. The two Lyapunov indexes of Cat mapping are

$$
L_{1}=\ln \left(\frac{3+\sqrt{5}}{2}\right)>0, \quad L_{2}=\ln \left(\frac{3-\sqrt{5}}{2}\right)<0 .
$$




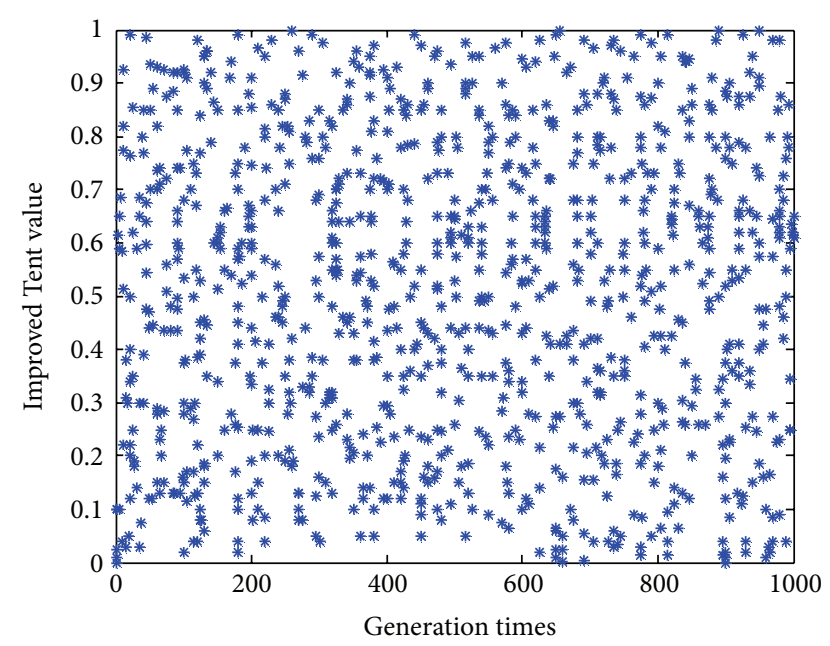

Figure 2: The chaos state of improved Tent mapping.

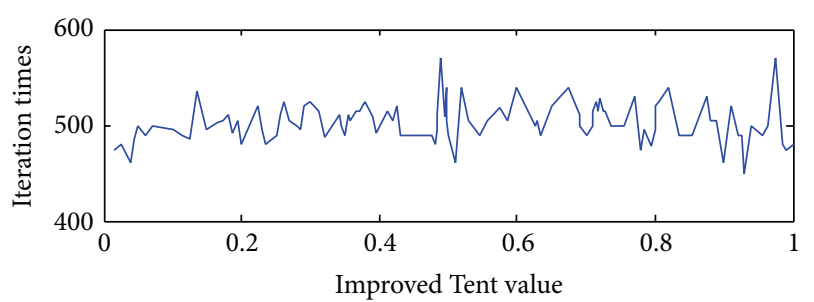

(a) Iterative distribution of improved Tent mapping

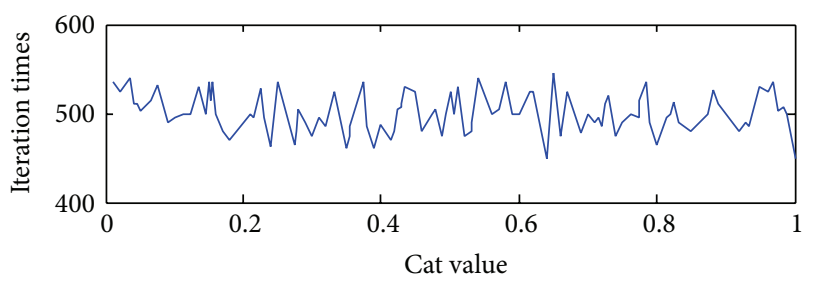

(b) Iterative distribution of Cat mapping

FIGURE 3: Two kinds of iterative distribution of mapping.

Thus the Cat map has the characteristics of chaotic. Figure 3 presents iterative distribution of improved Tent and Cat mapping.

We can see the Tent map and the Cat map are well distributed from Figure 3. The chaotic sequence is affected by the computer finite word length and accuracy. It can fall into a cycle quickly and converge after iteration. So the two chaotic sequences are suitable to generate initial population for genetic algorithm.

3.3. T-Shaped MIMO Radar Antenna Array Pattern Optimization Based on the Improved Chaotic Genetic Algorithm. This paper proposed used the Cat map to generate initial population and joined the chaotic disturbance using the improved Tent map. Chaos disturbance should be employed to individual of a generation, which is equal to conducting chaotic mutation to gene to reduce the evolving algebra of genetic algorithm. This can increase the diversity of the population and is likely to produce better gene sequences. It can not only improve search accuracy and speed, but also avoids effectively the problem of local convergence and prematurity of genetic algorithm.

We suppose corresponding relations between algorithm and antenna array parameters. Consider a MIMO radar antenna array consisting of $M$ transmitting antenna elements symmetrically distributed in $X$ axis and $N$ receiving antenna elements symmetrically distributed in $Y$ axis. The array element spacing, respectively, is $d_{x}, d_{y}$. The variables to be optimized $I_{t}, I_{r}$ are exciting current amplitude of transceiver array elements. Minimize the side-lobe level of T-shaped MIMO radar antenna as the optimization goal to achieve optimal array element excitation current amplitude.

Step 1 (generate initial population and encode). The initial population $\vec{I}(0)$ is generated randomly using (7) with $i=0$. The chromosome $I=\left[I_{t}, I_{r}\right]=\left[I_{1}, I_{2}, \ldots I_{M}, I_{M+1}, I_{M+2}\right.$, $\left.\ldots I_{M+N}\right]$ is made up together using real encoding, which is employed to the transmitting and receiving arrays of MIMO radar antenna. $P$ denotes the size of each population. $p_{c}$ denotes the crossover probability and $p_{m}$ denotes the mutation probability.

Step 2 (calculate the fitness value). Fitness function is used to measure each chromosome whether or not it can be better inheritance to the next generation. In order to reduce the side-lobe level of antenna array, we choose the fitness function as follows:

$$
\begin{gathered}
f=0.5 f_{1}+0.5 f_{2}, \\
f_{1}=\frac{1}{1+\left|\mathrm{BW}-\mathrm{BW}_{w}\right|}, \\
f_{2}=\frac{1}{1+\left[(1 / p) \sum_{\theta=1}^{p}|F(\theta)+30|^{2}\right]^{-1 / 2}},
\end{gathered}
$$

where BW is the main-lobe beam-width after optimization and $\mathrm{BW}_{w}$ is the expected main-lobe beam-width. $f_{2}$ represents the root-mean-square deviation of the maximum relative side-lobe level and the preset value $-30 \mathrm{~dB}$. $p$ is the sampling points in side-lobe area. Calculate the fitness value of every individual in the population. The larger the fitness value, the easier the inheritance to the next generation. The ideal optimal value of the fitness function $f$ is 1 .

Step 3 (judgment). Determine whether it is in line with the termination condition. If they meet the termination condition, computer could end the algorithm. Then, the system could find out the best individual from the population. Then jump to Step 4.

Step 4 (selection). According to the fitness value of each individual, we adopt the roulette method to select some excellent individuals from the $i$ th generation of $\vec{I}(i)$ to $\vec{I}(i+1)$ and generate new individuals instead of those which are not chosen.

Step 5 (crossing). The individuals of $\vec{I}(i)$ match in pairs randomly. And then they replace the parent individuals with 


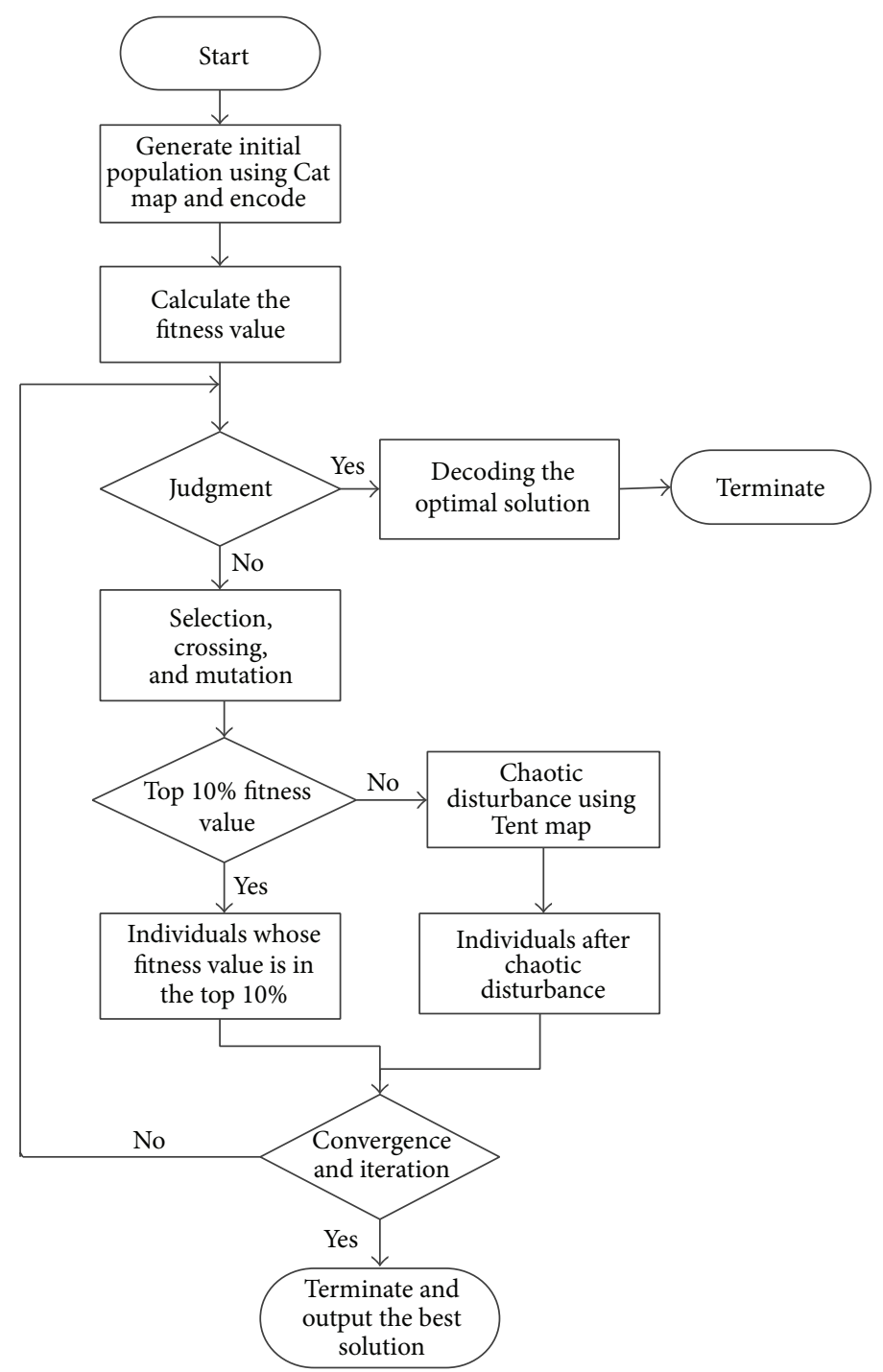

Figure 4: Flow chart for CGA processing.

crossover probability $p_{c}$ to restructure and generate new individuals.

Step 6 (mutation). Each individual in $\vec{I}(i)$ changes one or some genes with mutation probability $p_{m}$ and selected effective coding randomly instead of redundant code.

Step 7 (individuals whose fitness value is in the top $10 \%$ of mutated population $\vec{I}(i)$ do not do chaotic disturbance). But they will participate in the next genetic manipulation. Another $90 \%$ of the individuals do chaos disturbance. Using the iterative formula (7), we can calculate the chaotic vector $I_{i}^{\prime(k)}$ after $k$ iterations, where $k$ is used to identify the number of iterations in chaotic sequence.

Step 8. Screen vectors after chaotic disturbance and calculate the new fitness value $f^{\prime}$.

Step 9. Judge the convergence of the fitness value of population $\vec{I}(i)$. If the computational result is convergent or the iteration number is equal to the designed maximum iteration number, the algorithm will be terminated and output the best solution; else, jump to Step 4.

\section{Simulated Results and Discussion}

For a T-shaped MIMO radar antenna array with $d=1.2 \lambda$, $M=N=10$, we request the main-lobe point at $\theta=0^{\circ}$ and the feed phase of each transmitting and receiving array is 0 . We optimize array element excitation current amplitude of transceiver antenna using the improved chaos genetic algorithm in this paper. Under the condition of expected main-lobe beam-width, we can get side-lobe level as low as possible. We set parameters $P=100, p_{c}=0.8$, and $p_{m}=0.05$. Iteration step is 100 . The expected main lobe beam-width is $10^{\circ}$. Figure 5 presents the normalized pattern of antenna array before and after optimization using algorithm in this paper (CGA) and the traditional genetic algorithm (GA). The two 
TABLE 1: Simulation results of the algorithms.

\begin{tabular}{lcccc}
\hline Algorithm & Average of SLL $(\mathrm{dB})$ & Average fitness & Best case of SLL $(\mathrm{dB})$ & Worst case of SLL $(\mathrm{dB})$ \\
\hline GA & -33.7 & 0.84 & -39.6 & -30.7 \\
CGA & -41.5 & 0.97 & -50.8 & -38.2 \\
\hline
\end{tabular}

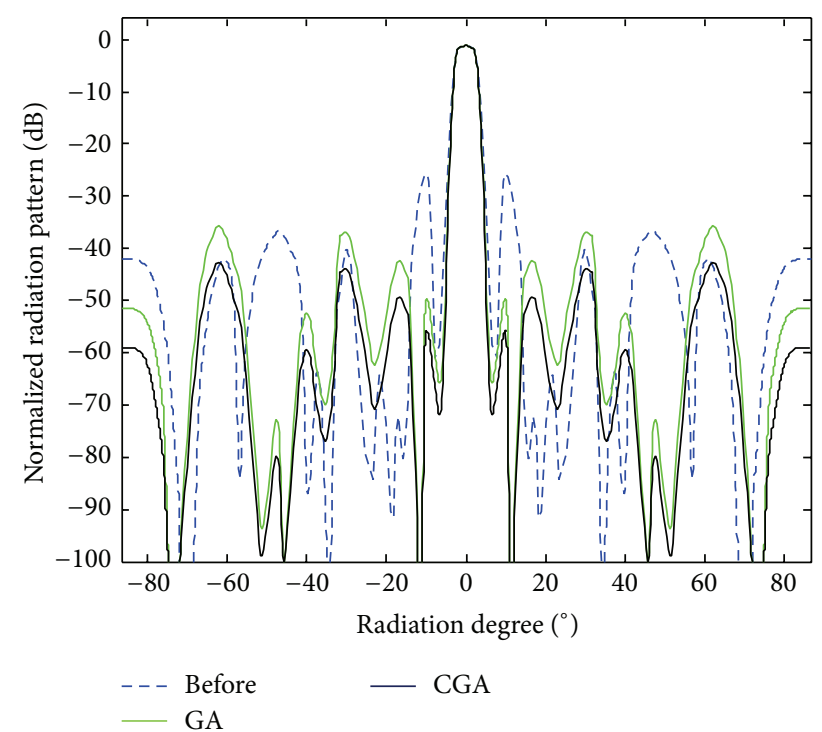

FIGURE 5: The normalized pattern used improved chaotic genetic algorithm and the traditional genetic algorithm.

algorithms can satisfy the main-lobe beam-width with $10^{\circ}$. The maximum relative side-lobe level is $-33 \mathrm{~dB}$ and the first side-lobe level is $-50 \mathrm{~dB}$ using GA. The maximum relative side-lobe level is $-43 \mathrm{~dB}$ and the first side-lobe level is $-55 \mathrm{~dB}$ using CGA, which is much better than the corresponding values using GA. Figure 6 shows the convergence curves of objective function of the two algorithms.

In order to analyze the optimization effect for the sidelobe levels (SLL) by the algorithms, 50 simulations have been done by GA and CGA, respectively. The results of the simulations are listed in Table 1. From Table 1, we can see that the CGA result of the average of SLL $(-41.5 \mathrm{~dB})$ is lower than that of GA $(-33.7 \mathrm{~dB})$. So the algorithm in this paper is superior to the traditional genetic algorithm.

\section{Conclusion}

In this paper, a new improved chaos genetic algorithm is presented and applied to synthesize T-shaped MIMO radar array antenna pattern by controlling array element excitation current amplitudes. An improved Tent map and a novel twodimension Cat chaotic mapping have been put forward. We present the improved chaos genetic algorithm to reduce the maximum relative side-lobe level of antenna array. The novel algorithm effectively solves the design problems of MIMO radar antenna pattern synthesis in low side-lobe level. The simulation results verify the feasibility and effectiveness of the algorithm and make comparison with the traditional genetic

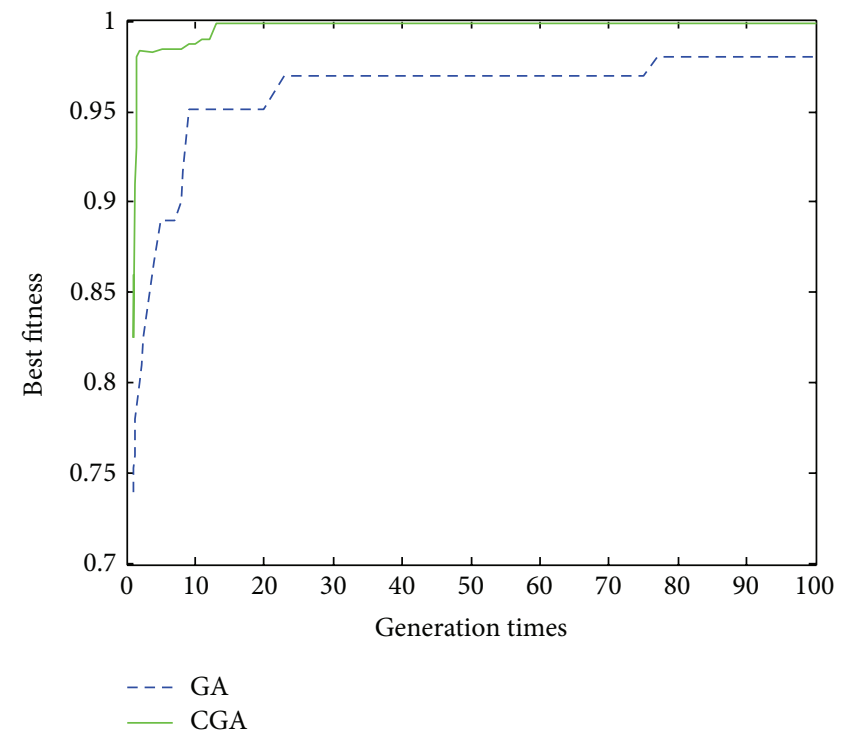

FIGURE 6: The convergence curves of objective function.

algorithm. From the simulation results we can see that the algorithm in this paper is superior to the traditional genetic algorithm. The improved chaotic genetic algorithm has a good application prospect to solve the problem of antenna array.

\section{Conflict of Interests}

The authors declare that there is no conflict of interests regarding the publication of this paper.

\section{Acknowledgments}

This work is partially supported by the National Natural Science Foundation of China under Grant no. 61333002.

\section{References}

[1] G. Xie, J. L. Gao, and K. M. Xie, "Fuzzy modeling based on rough sets and mind evolutionary algorithm," Journal of Electronic Measurement and Instrument, vol. 1, no. 8, pp. 109-112, 2003.

[2] Y. Fan, R.-H. Jin, and B. Liu, "Study on the objective function for the array pattern synthesis based on genetic algorithm," Journal of Electronics and Information Technology, vol. 27, no. 5, pp. 801804, 2005.

[3] X. S. He, W. J. Ding, and X. S. Yang, "Bat algorithm based on simulated annealing and Gaussian perturbations," Application Research of Computers, vol. 31, no. 3, pp. 392-397, 2013.

[4] W. P. Liao and F. L. Chu, "Array pattern synthesis with null steering using genetic algorithms by controlling only the current 
amplitudes," International Journal of Electronics, vol. 86, no. 4, pp. 445-457, 1999.

[5] J. F. Ye, Research on Antenna Array Synthesis of Immune Algorithm, Harbin Engineering University, 2009.

[6] L. N. de Castro and F. J. von Zuben, "Learning and optimization using the clonal selection principle," IEEE Transactions on Evolutionary Computation, vol. 6, no. 3, pp. 239-251, 2002.

[7] L. Cen, Z. L. Yu, W. Ser, and W. Cen, "Linear aperiodic array synthesis using an improved genetic algorithm," IEEE Transactions on Antennas and Propagation, vol. 60, no. 2, pp. 895-902, 2012.

[8] L. Cen, W. Ser, Z. L. Yu, and S. Rahardja, "An improved genetic algorithm for aperiodic array synthesis," in Proceedings of the IEEE International Conference on Acoustics, Speech and Signal Processing (ICASSP '08), pp. 2465-2468, Las Vegas, Nev, USA, April 2008.

[9] H. Z. Xue and W. M. Li, Antenna Array Analysis and Synthesis, Beijing University of Areonautics and Astronautics press, 2011.

[10] R. J. Mailloux, Phased Array Antenna Handbook, Artech House, Boston, Mass, USA, 1993.

[11] R. C. Johnson, Antenna Engineering Handbook, McGraw-Hill, New York, NY, USA, 3rd edition, 1993.

[12] M. Ridwan, M. Abdo, and E. Jorswieck, "Design of nonuniform antenna arrays using genetic algorithm," in Proceedings of the 13th International Conference on Advanced Communication Technology: Smart Service Innovation through Mobile Interactivity (ICACT '11), pp. 422-427, IEEE, Seoul, Republic of Korea, February 2011.

[13] X. Che and L. Bian, "Notice of retraction low-side-lobe pattern synthesis of array antennas by genetic algorithm," in Proceedings of the 4th International Conference on Wireless Communications, Networking and Mobile Computing (WiCOM '08), pp. 1-4, 2008.

[14] M. O. Binelo, A. L. F. De Almeida, and F. R. P. Cavalcanti, "MIMO array capacity optimization using a genetic algorithm," IEEE Transactions on Vehicular Technology, vol. 60, no. 6, pp. 2471-2481, 2011.

[15] Z. Wang, Z. He, J. Hu, and J. Xie, "A low side-lobe array weighted optimization method in sky-wave radar," in Proceedings of the 5th IEEE International Symposium on Phased Array Systems and Technology (ARRAY '13), pp. 379-382, Waltham, Mass, USA, October 2013.

[16] S. Li, "Grate lobes/side lobes suppression for sparse array design by using genetic algorithms," in Proceedings of the 2nd International Conference on Innovations in Bio-inspired Computing and Applications (IBICA '11), pp. 371-373, Shenzhan, China, December 2011.

[17] J. Yuan, H. Zhou, C. Guo, and J. Ding, "Efficient optimization of shaped-beam sparse linear antenna arrays using genetic algorithm," in Proceedings of the International Conference on Microwave and Millimeter Wave Technology, pp. 1-4, IEEE, May 2012.

[18] L. Shan, H. Qiang, J. Li, and Z.-Q. Wang, "Chaotic optimization algorithm based on Tent map," Control and Decision, vol. 20, no. 2, pp. 179-182, 2005.

[19] Y. Wang, L. Liu, B. Zhou, and H. Zhang, "Detection method of printed circuit board components based on chaotic optimization algorithm," Chinese Journal of Scientific Instrument, vol. 31, no. 2, pp. 410-415, 2010.

[20] A. El-Gohary and A. S. Al-Ruzaiza, "Chaos and adaptive control in two prey, one predator system with nonlinear feedback," Chaos, Solitons and Fractals, vol. 34, no. 2, pp. 443-453, 2007.
[21] A. Lommi, A. Massa, E. Storti, and A. Trucco, "Sidelobe reduction in sparse linear arrays by genetic algorithms," Microwave and Optical Technology Letters, vol. 32, no. 3, pp. 194-196, 2002.

[22] D. P. Tian, "Particle swarm optimization algorithm based on tent chaotic sequence," Computer Engineering, vol. 36, no. 4, pp. 180-182, 2010.

[23] X. H. Yang, Q. Chen, and F. Z. Yang, "Chaos higher efficient genetic algorithm for the forecast of the sediment concentration in reservoir," Journal of Beijing Normal University, vol. 43, no. 2, pp. 194-198, 2007.

[24] F. C. Robey, S. Coutts, D. Weikle, J. C. McHarg, and K. Cuomo, "MIMO radar theory and experimental results," in Proceedings of the 38th Asilomar Conference on Signals, Systems and Computers, pp. 300-304, IEEE, November 2004.

[25] L. Shan, H. Qiang, J. Li, and Z. Q. Wang, "Chaotic optimization algorithm based on Tent map," Control and Decision, vol. 20, no. 2, pp. 179-182, 2005.

[26] F. Wang, S. Y. Dai, and S. S. Wang, "Modified chaos-genetic algorithm," Computer Engineering and Applications, vol. 46, no. 6, pp. 29-32, 2010. 

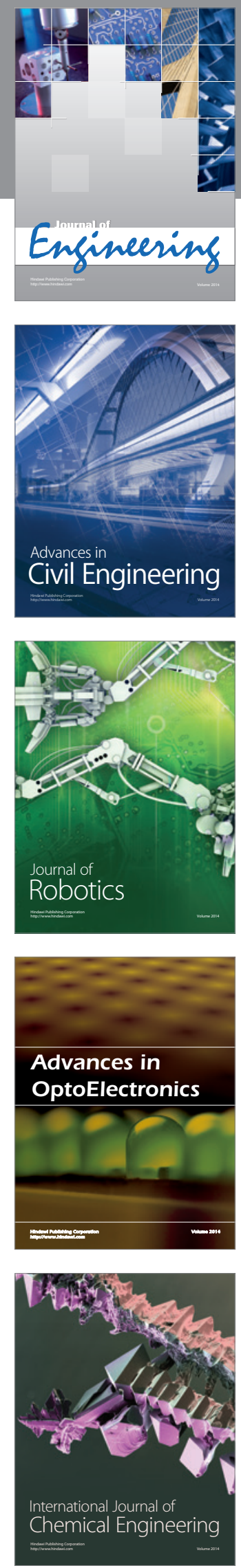

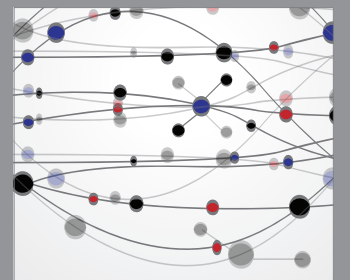

The Scientific World Journal
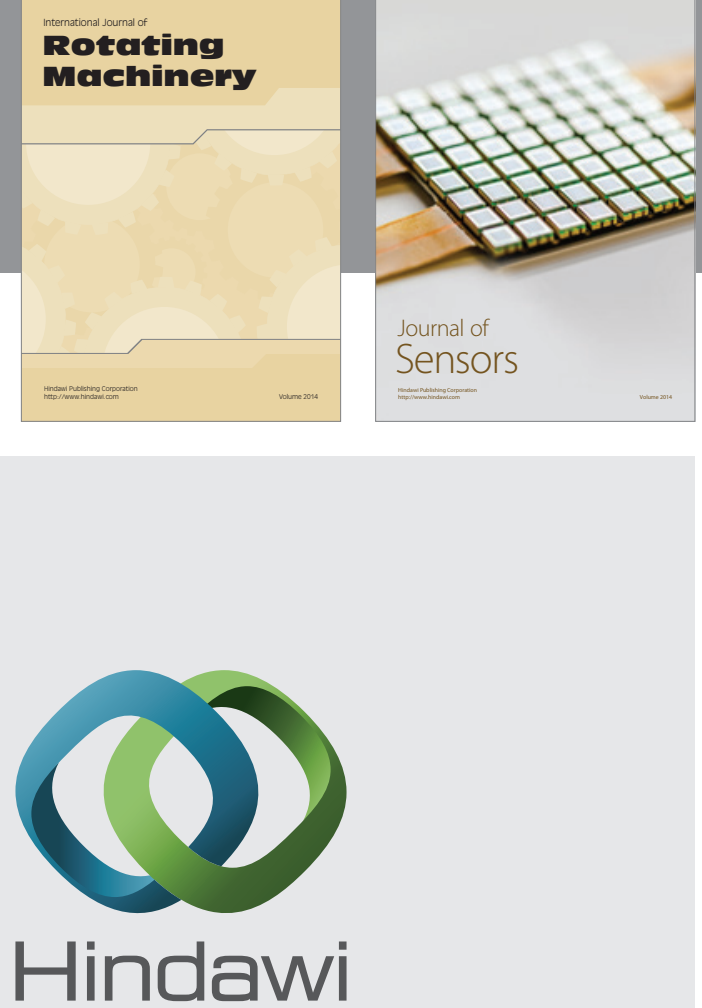

Submit your manuscripts at http://www.hindawi.com
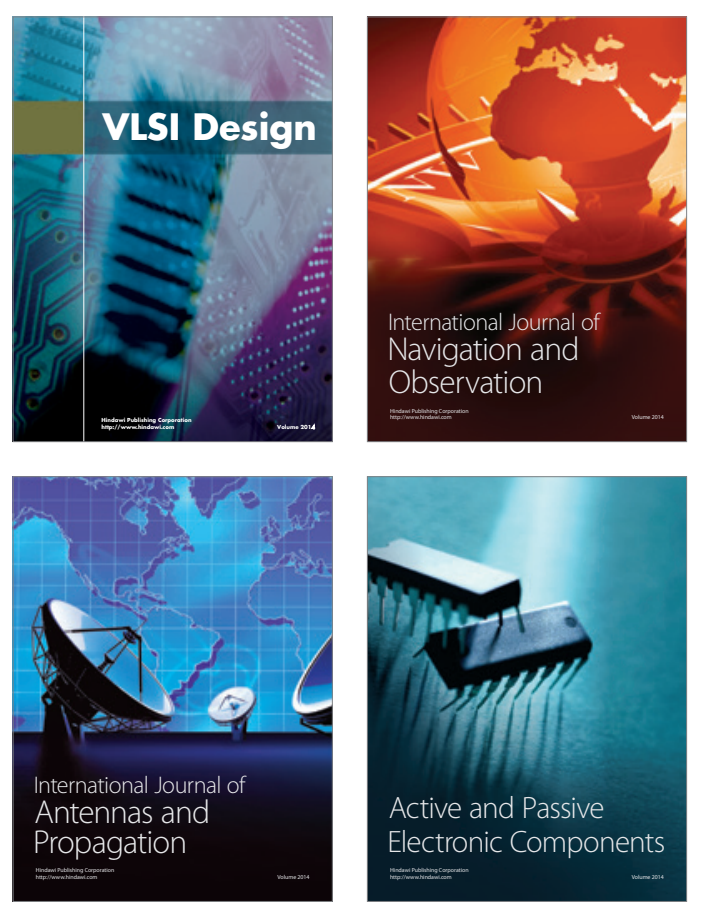
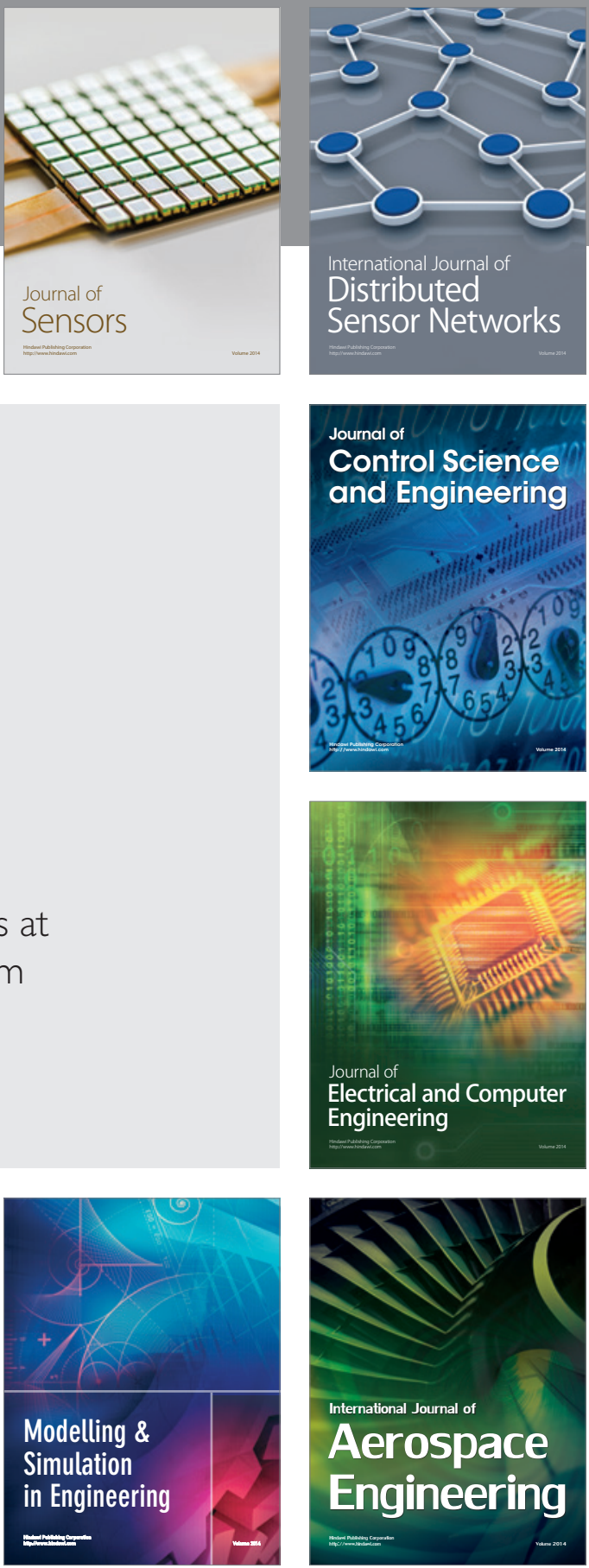

Journal of

Control Science

and Engineering
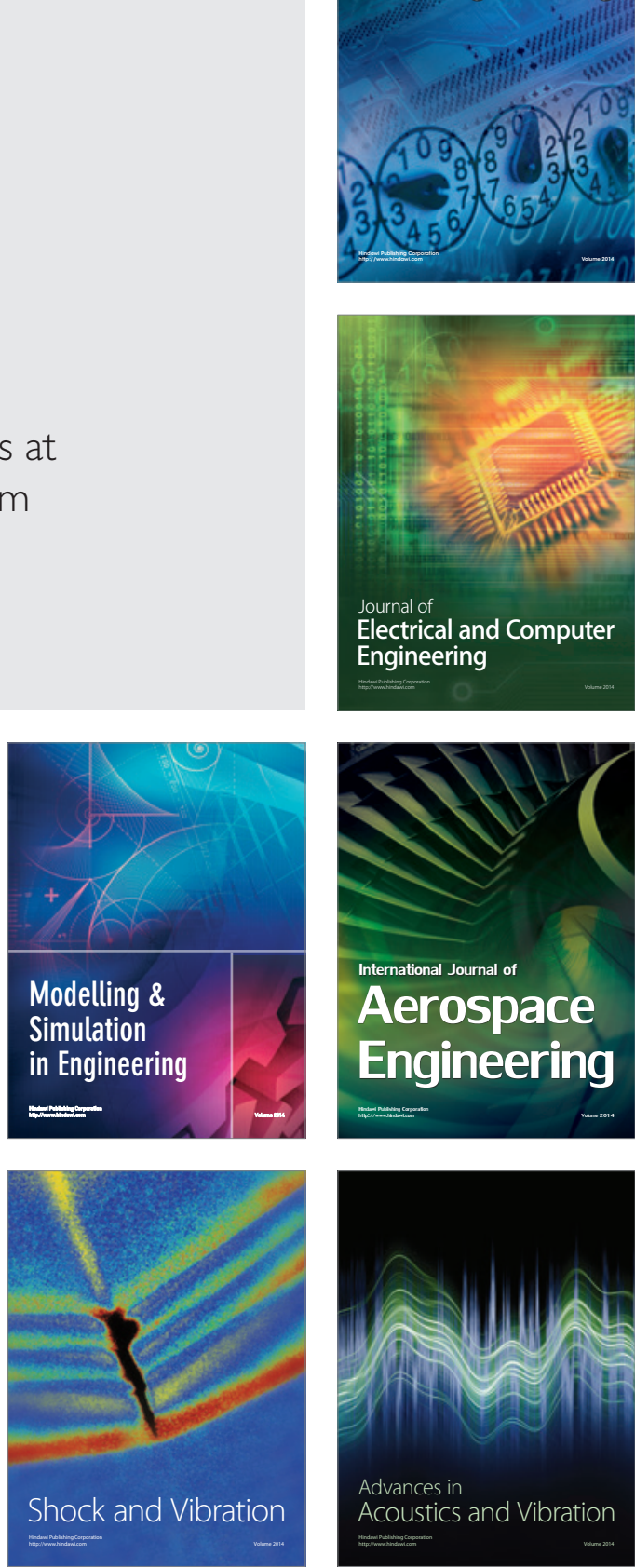